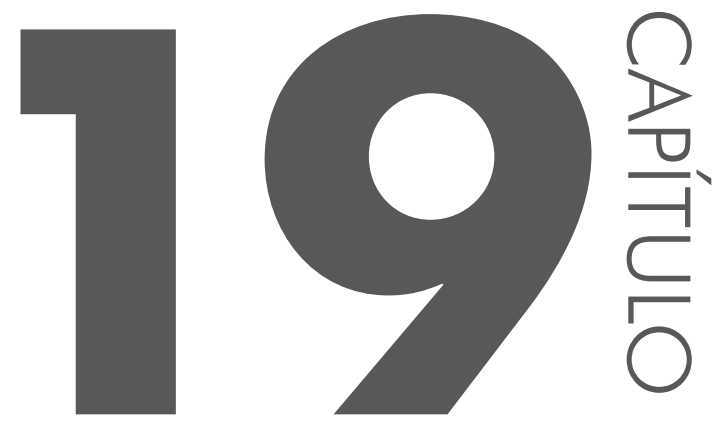

\title{
REVISÃO DE HEURÍSTICAS PARA MINIMIZAÇÃO DO TEMPO DE FLUXO EM SISTEMAS FLOW SHOP COM TEMPOS DE SETUP
}

Fernanda Neiva Mesquita ${ }^{1}$ Hélio Yochibiro Fuchigami ${ }^{1}, 2$

Resumo: Este trabalho aborda problemas de programação em flow shop para minimização do tempo de fluxo (flow time) com tempos de setup dependente

1 Unidade Acadêmica Especial de Gestão e Negócios, Universidade Federal de Goiás - Regional Catalão, Catalão, Brasil.

2 Campus Aparecida de Goiânia, Universidade Federal de Goiás, Aparecida de Goiânia, Brasil. E-mail de contato: nandi_mesquita@hotmail.com, heliofuchigami@yahoo.com.br 
ou independente da sequência das tarefas, cujo método de solução são as heurísticas. Foram analisados artigos que tratam do tema, observando características em comuns e/ou predominantes, possíveis lacunas e potenciais de pesquisa. $\mathrm{O}$ estudo mostra a predominância da soma dos tempos de fluxo das tarefas, dentre as variações possíveis para as medidas de desempenho relacionadas ao fluxo de produção, e também a maior quantidade de trabalhos abordando problemas com duas máquinas.

Palavras-chave: Flow shop. Tempos de setup. Flow time. Heurística.

Abstract: This paper focuses on programming problems in shop flow to minimize the flow of time with setup times dependent or independent of the sequence of tasks, whose solution method are the heuristics. Articles were analyzed on the same matter, observing characteristics in common and/or predominant, possible gaps and potential for research. The study shows the prevalence of the sum of the tasks flow times, among the possible variations for the performance measures related to the production flow, and also the largest number of works addressing problems with two machines.

Keywords: Flow shop. Time setup. Flow time. Heuristic.

\section{INTRODUC̣ÃO}

O mercado busca por produtos que atendam a algumas características como o preço, a qualidade e também o prazo de entrega. Desta forma, a gestão da produção tem papel de gerenciar os recursos por meio de uma produção mais enxuta, com vistas ao atendimento destes clientes de forma eficiente para fidelizar e atrair cada vez mais consumidores. Dessa maneira, geralmente, o número de programações possíveis cresce rapidamente à medida que o número de atividades e processos aumenta (ANDRADE, 1990; CORRÊA; CORRÊA, 2005).

A atividade de programação é uma das mais complexas tarefas no gerenciamento de produção. Primeiro, os programadores podem lidar com diversos tipos diferentes de recursos simultaneamente. As máquinas podem ter diferentes capacidades e o pessoal, distintas habilidades. De maneira mais importante, geralmente, o número de programações possíveis cresce rapidamente à medida que o número de atividades e processos aumenta (SLACK et al., 1999; ARENALES et al., 2007).

A programação da produção corrobora para a boa ordenação das tarefas que serão executadas; para isso, considera-se uma fração de tempo e a quantidade de máquinas alocadas, ou seja, trata-se da determinação de local e período de execução de cada operação, o que otimizará o processo produtivo (LAWLER, 1989; BAKER;TRIETSCH, 2009). Quanto à metodologia dessa pesquisa, trata-se 
de uma análise bibliográfica, que, de acordo com Vergara (1998), é desenvolvida com o objetivo de proporcionar uma visão geral, de tipo aproximativo, acerca de determinado fato. Este tipo de pesquisa é realizado especialmente quando o tema escolhido é pouco explorado e torna-se difícil sobre ele formular hipóteses precisas e operacionais.

O trabalho se propõe a analisar ambientes de produção do tipo flow shop, caracterizado pelo fato de os materiais e peças se deslocarem na fábrica com rotas constantes e com alto grau de automação, capazes de produzir um restrito grupo de produtos em grandes quantidades e a baixos custos. Os processos e as operações estão balanceados para um pequeno grupo de produtos, reduzindo a necessidade de estoques intermediários, que são muito pequenos quando comparados aos estoques de produtos acabados (HAX; CANDEA, 1984).

O flow shop contém $m$ máquinas, e as ordens compõem-se de sequências de operações estritamente ordenadas. Todos os movimentos devem ter uma direção uniforme. Um exemplo é uma linha de montagem com estações de trabalho compostas de uma máquina. (HAX; CANDEA, 1984, p. 268)

Um gasto despendido, que será considerado na pesquisa, é o tempo de setup, que constitui os tempos reservados na preparação das máquinas, as quais receberão as tarefas. Quando o setup depende apenas da tarefa que espera por processamento, é considerado independente e quando o setup também depende da tarefa que foi processada anteriormente na máquina, é considerado dependente (ZACCARELLI,1987).

A medida de desempenho a ser minimizada é o tempo de fluxo (flow time) que equivale ao tempo que uma tarefa gasta desde o instante em que está liberada até o final do seu processamento, ou seja, é o tempo que uma tarefa permanece na máquina (TAHA, 2008).

Um problema de produção é denotado na literatura específica por meio de uma notação matemática de três campos. Pinedo (2012) organiza esses parâmetros em um trio $\alpha|\beta| \gamma$ que auxilia a classificação de um problema de sequenciamento. Esses campos determinam o problema, descrevendo $\alpha$ como o ambiente de produção com o número de máquinas ou estágios, $\beta$ fornecendo detalhes das limitações dos recursos e tarefas, e $\gamma$ que contém o objetivo a ser melhorado, ou seja, a medida de desempenho.

De acordo com essa conhecida notação de três campos, o problema pode ser representado pelas seguintes formas: $F\left|s_{j k}\right| \sum F_{j}, F\left|s_{j k}\right| \sum F_{j}, F\left|s_{j k}\right| \bar{F}, F\left|s_{i j k}\right| \bar{F}$, $F\left|s_{j k}\right| \sum \alpha F_{j}$ ou $F\left|s_{i j k}\right| \sum \alpha F_{j}$ onde $F$ representa o ambiente de produção em flow shop, as restrições do problema denotado pelo $s_{\mathrm{jk}}$ que é a presença de tempos de setup independentes da sequência e $s_{i j k}$ que é a presença de tempos de setup de- 
pendentes da sequência. O campo $\sum F_{j}, \bar{F}, \sum \alpha F_{j}$ define a medida de desempenho considerada, ou seja, tempo de fluxo flow time.

Uma observação importante é que outras restrições, além dos tempos de setup, foram citadas à medida que nos artigos pesquisados os autores considerassem nos problemas. Existem diversas técnicas clássicas de resolução, tais como método branch-and-bound, simplex, relaxação lagrangeana, métodos heurísticos, dentre outros. Pode ser muito bem aplicada em sistemas logísticos de planejamento e controle da produção (SOUZA; MOCCELLIN, 2000; CORREA; GIANESI; CAON, 2006).

Restringiu se a revisão bibliográfica por métodos de solução heurísticos, pois buscam critérios racionais, com fácil implementação, não exigindo altos níveis computacionais, em que se chega numa solução viável pelo menos próxima da ótima com tempos de computação aceitável. Assim, o presente estudo se propõe a realizar uma análise da literatura, buscando as características comuns e/ou predominantes, as lacunas para futuras pesquisas e as potenciais áreas ainda pouco exploradas, tratando de minimização do flow time (médio, total ou ponderado) e fluxo das tarefas nas máquinas em flow shop, com presença de setup dependente ou independente, que utilizaram heurísticas como método de resolução.

\section{REVISÃO DA LITERATURA}

Nesta pesquisa, foi realizada uma busca nas bases de dados de periódicos científicos utilizando se das seguintes palavras-chave: "flow shop", "flow time" e "setup". Delimitando posteriormente aos problemas de flow shop com tempos de setup dependente ou independente com minimização do flow time, além disso, restringiu-se ainda a métodos heurísticos. É importante ressaltar que foram citados todos os artigos encontrados até o momento, naturalmente podendo não contemplar alguns artigos existentes na literatura.

Foram encontrados na pesquisa, artigos de revisão bibliográfica, mas que não contemplava a restrição de tempos de setup. Como o artigo de Pan e Ruiz (2013) no qual abrange uma revisão e evolução computacional, assim como realiza uma avaliação estatística de 22 heurísticas existentes. Para a compreensão, obteve-se assim uma detalhada comparação de cinco novas heurísticas, aplicando-se a técnica da ANOVA. A comparação dos resultados identificou os melhores métodos existentes e mostrou que cinco novas heurísticas presentes eram competitivas, ou possuem melhor performance que alguns problemas de flow shop permutacional com flow time da literatura.

Outra revisão recente do tema, sem considerar os tempos de setup, foi desenvolvida por Fernandez-Viagas e Framinan (2015), que propuseram uma nova heurística de complexidade para o problema de flow shop permutacional com 
minimização do flow time, obtiveram melhores resultados do que a heurística LR proposta por Liu e Reeves (2001). Os autores replicaram a heurística LR obtendo uma nova a partir de 17 heurísticas eficientes para o problema, com 15 delas incorporando propostas dos autores. Os autores também discutiram algumas questões relativas à evolução das heurísticas eficientes para o problema e propuseram indicadores alternativos.

O problema mais antigo encontrado foi o de Rajendran e Ziegler (1997), que tratam de setup dependente das tarefas e propuseram uma nova heurística para minimizar o flow time ponderado. Os autores Aldowaisan e Allahverdi (1998) associaram ao problema a restrição de sem espera entre as operações das tarefas e setup dependente para duas máquinas. Posteriormente, Allahverdi (2000) descreveram diferentes classes de tarefas com setup independentes, resolvendo com heurísticas e comparando com o método ótimo de branch-and-bound.

Aldowaisan (2001), baseado no artigo de Aldowaisan e Allahverdi (1998), aplicou uma nova heurística ao problema tratado. Allahverdi e Aldowaison (2001) criaram heurísticas com satisfatórias demonstrações de experiências computacionais utilizando o conceito de repetição de inserção no algoritmo.

Posteriormente, Allahverdi, Aldowaisan e Sotskov (2002) utilizaram uma distribuição livre de tempos de setup para calcular o limite inferior e superior. Framinan, Leisten e Ruiz-Usano (2005) propuseram uma comparação e classificação das heurísticas existentes e depois criaram duas novas heurísticas para o problema de flow shop permutacional. Allahverdi (2006) realizou experimentos computacionais mostrando a relação de dominação desenvolvida no problema e uma forma de como reduzi-la.

Pesquisaram Wang e Cheng (2005) o problema de flow shop com duas máquinas propondo heurísticas e compararamos resultados com o método de branch-and-bound. Ladhari, Msakni e Allahverdi (2012) estudaram uma nova regra de prioridade para várias heurísticas construtivas e compararam os resultados com o algoritmo genético de busca local. Gharbi et al. (2013) criaram lower bounds para minimização do tempo total de fluxo baseado na relaxação lagrangeana. Enquanto Nagano, Miyata e Araujo (2014) desenvolveram várias heurísticas com restrição de sem espera entre as operações das tarefas com tempos de setup dependente.

\subsection{Análise da literatura}

A Figura 1 apresenta a organização dos trabalhos revisados em ordem cronológica e a relação existente entre eles. As setas com linhas cheias indicam quando um trabalho serviu como base para o desenvolvimento de outro e as setas tracejadas quando um artigo comparou o seu método com outro para avaliar o resultado. 


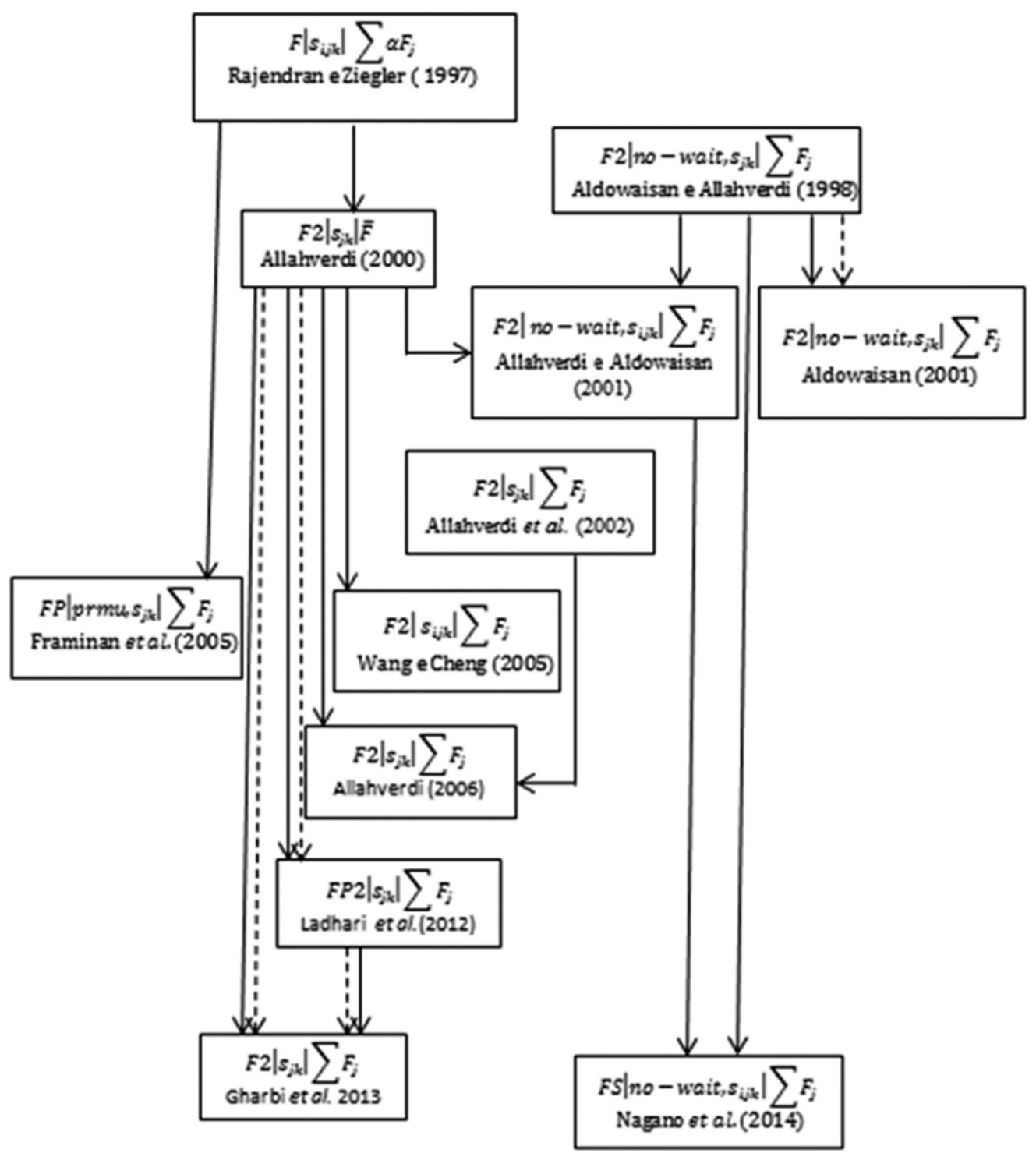

Legenda

no- wait: program ação sem tem po de espera entre as operações das tarefas.

prmu: a solução deve ser um a program ação perm utacional. $\alpha$ : peso

Figura 1. Representação em ordem cronológicas dos artigos de flow shop, com tempos de setup dependente ou independente e minimização do flow time. 
Além da representação visual, os trabalhos citados também foram organizados de acordo com a Tabela 1, que inclui o método de solução utilizado no problema. Dentre os 12 artigos analisados, como mostra a Tabela 1, todos utilizaram como método de solução as heurísticas. Alguns somente usaram as heurísticas outros em paralelo com outros métodos, com objetivo de comparar os resultados.

Tabela 1. Descrição dos artigos citados na Figura 1 e seus respectivos métodos de solução

\begin{tabular}{|c|c|c|}
\hline Autores/Ano & Notação & Método de Resolução \\
\hline Rajendran e Ziegler ( 1997) & $F\left|s_{i j}\right| \sum \alpha F_{j}$ & Heuristica \\
\hline Aldowaisan e Allahverdi (1998) & $F 2 \mid$ no-wait, $s_{i j} \mid \sum F_{j}$ & Heuristica \\
\hline Allahverdi $(2000)$ & $F 2\left|s_{j}\right| \bar{F}$ & Heuristical Branch-and-bound \\
\hline Aldowaisan (2001) & $F 2 \mid$ no-wait, $s_{j} \mid \sum F_{j}$ & Heuristica \\
\hline Allahverdi e Aldowaisan (2001) & $F 2 \mid$ no-wait, $s_{i j k} \mid \sum F_{j}$ & Heuristica \\
\hline Allahverdi et al (2002) & $F 2\left|s_{j k}\right| \sum F_{j}$ & Heuristica \\
\hline Allahverdi (2006) & $F 2\left|s_{j k}\right| \sum F_{j}$ & Heuristica \\
\hline Wang e Cheng (2005) & $F 2\left|s_{i j}\right| \sum F_{j}$ & Heuristical Branch-and-bound \\
\hline Framinan et al. (2005) & $F P\left|p r m u, s_{j}\right| \sum F_{j}$ & Heuristica \\
\hline Ladhari et al. (2012) & $F P 2\left|s_{j k}\right| \sum F_{j}$ & $\begin{array}{c}\text { Heuristicas/Algoritmo genético de } \\
\text { busca local }\end{array}$ \\
\hline Gharbi et al. (2013) & $F 2\left|s_{j k}\right| \sum F_{j}$ & Heuristica/ Relaxação Lagrangea \\
\hline Nagano et al.(2014) & $F \mid$ no-wait,,$s_{i j} \mid \sum F_{j}$ & Heuristica \\
\hline
\end{tabular}

Analisando os dados encontrados na Figura 1, o estudo mostra uma característica comum, que é a predominância da soma dos tempos de fluxo das tarefas, dentre as variações possíveis para as medidas de desempenho relacionadas ao fluxo de produção e também a maior quantidade de trabalhos abordando 
problemas com duas máquinas. Não foram encontrados trabalhos que fizessem comparações heurísticas e outro método ótimo, além do branch-and-bound. Os demais métodos de solução foram a utilização do algoritmo genético modificado e a relaxação lagrangeana.

Foram doze problemas tratando de flow shop, dentre esses oito eram para o caso de duas máquinas e um caso de flow shop permutacional e flow shop permutacional com duas máquinas. A restrição de tempos de setup foram nove dependentes e o restante para casos de setup independentes.

O tempo de espera da tarefa entre as máquinas foi considerado nos artigos de Aldowaisan e Allahverdi (1998), Aldowaisan (2001), Allaverdi e Aldowaisan (2001) e Nagano et al. (2014), aplicando a restrição no-wait sem tempo de espera entre as operações das tarefas.

Quanto à medida a ser minimizada do tempo de fluxo, 10 artigos utilizaram a soma do tempo total de fluxo e os demais, como Rajendran e Ziegler (1997 utilizaram o tempo total de fluxo ponderado e Allahverdi (2000) o tempo médio de fluxo. O artigo de Allahverdi (2000) foi o que mais serviu como base para o desenvolvimento de outros artigos e também o que outros autores mais compararam o seu método para avaliar seus resultados. Nota-se ainda que os estudos foram feitos de forma mais independente uns dos outros, não estabelecendo uma hierarquia, tanto em termos de elaboração de métodos como em comparações de resultados.

Uma fragilidade da evolução dos métodos está justamente no fato de não comparar seus resultados com pesquisas anteriores. Isto ocorreu principalmente nos anos iniciais das publicações, provavelmente pela dificuldade em se acessar os trabalhos. Nas décadas mais recentes é possível que o maior acesso tenha viabilizado as comparações dos resultados dos métodos.

Os problemas avaliados não contêm diferentes restrições, mostrando a tendência de não se considerar situações mais realistas. Isto acarreta o tratamento de problemas mais fáceis e menor número de restrições práticas. Um potencial de pesquisa nesse campo seria considerar novas restrições realísticas aos problemas, ou mesmo propor novas heurísticas comparando as já existentes.

\section{CONCLUSÃO}

A revisão bibliográfica contribuiu com um exame minucioso da literatura e apresentou o estado da arte dos artigos encontrados até o momento, descreveu a evolução do problema tratado. Foi possível organizar a literatura de forma visual e também realizar uma análise das publicações e a esquematização os artigos em forma de árvore. 
Pode se perceber um baixo número de artigos que tratam de flow shop com tempos de setup tanto dependente como independe, bem como os que utilizam como medida de desempenho para minimizar o problema as heurísticas.

Para novas pesquisas, propõe-se estudo de novos métodos de solução para os problemas mais recentes e comparação dos resultados. Além de pesquisar as restrições mais realísticas que ainda não foram estudadas e adaptar os métodos de artigos anteriores para validar a solução obtida.

\section{REFERÊNCIAS}

ALDOWAISAN, T. A.; ALLAHVERDI, A. Total flow time in no-wait flow shops with separated setup times. Computers and Operations Research, v. 25, n. 9, p. 757-765, 1998. ALDOWAISAN, T. A. Minimizing mean flow time in a two-machine flow shop with sequence-independent setup times. Computers and Operations Research, v. 49, p. 10011006, 2001.

ALLAHVERDI, A. A new heuristic and dominance relations for no-wait flow shops with setups minimizing mean flow time in a two-machine flow shop with sequence independent setup times. Computers and Operations Research, v. 27, p. 111-127, 2000. . Two-machine flowshop scheduling problem to minimize total completion time with bounded setup and processing times. The International Journal of Production Economics, v. 103, p. 386-400, 2006.

ALLAHVERDI, A.; ALDOWAISAN, T. Minimizing total completion time in a nowait flowshop with sequence-dependent additive changeover times. Computers and Operations Research, v. 52, p. 449-462, 2001.

ALLAHVERDI, A.; ALDOWAISAN, T.; SOTSKOV, Y. N. Two-machine flow shop scheduling problem to minimize make span or total completion time with random and bounded setup times. The International Journal of Management Science, v. 39, p. 2475 2486, 2002.

ANDRADE, E. L. Introdução à pesquisa operacional. Rio de Janeiro: LTC, 1990.

ARENAleS, M.; ARMENTANO, V.; MORABITO, R.; YANASSE, H. Pesquisa operacional para cursos de engenharia. São Paulo: Campus, 2007.

BAKER, K. R. ; TRIETSCH, D. Principles of sequencing and scheduling. Nova Jersey: John Wiley \& Sons, 2009.

CORRÊA, H. L.; CORRÊA, C. A. Administração da produção e de operações: manufatura e serviços: uma abordagem estratégica. Edição compacta. São Paulo: Atlas, 2005.

CORREA, H. L. GIANESI, I. G. N.; CAON, M. Planejamento, programação e controle da produção. São Paulo: Atlas, 2006.

FERNANDEZ-VIAGAS, V.; FRAMINAN, J. M. A new set of high-performing heuristics to minimise flow time in permutation flow shops. Computers \& Operations Research, v. 53, p. 68-80, 2015. 
FRAMINAN, J. M.; LEISTEN, R.; RUIZ-USANO, R. Comparison of heuristics for flow time minimisation in permutation flow shops. Computers and Operations Research, v. 32, p. 1237-1254, 2005.

GHARBI, A.; LADHARI, T.; MSAKNI, M. K.; SERAIRI, M. The two-machine flow shop scheduling problem with sequence-independent setup times: New lower bounding strategies, European Journal of Operational Research, v. 231, p. 69-78, 2013.

HAX, A. C.; CANDEA, D. Production and inventory management. Nova Jersey: Prentice Hall, 1984.

LADHARI, T.; MSAKNI, M. K.; ALLAHVERDI, A. Minimizing the total completion time in a two-machine flow shop with sequence-independent setup times, Journal of the Operational Research Society, v. 63, p. 445-459, 2012.

LAWLER, E. Production and inventory management. Houston: American Productivity and Quality Conter, 1989.

LIU, J. Y.; REEVE, C. R. Constructive and composite heuristic solutions to the P// $\sum \mathrm{C}_{\mathrm{i}}$ scheduling problem, European Journal of Operational Research, v. 132, p. 439-452, 2001. NAGANO, M. S.; MIYATA, H. H., ARAUJO, D. C. A constructive heuristic for total flow time minimization in a no-wait flow shop with sequence dependent setup times, Journal of Manufacturing Systems, v. 36, p. 224-230, 2014.

PAN, Q.-K.; RUIZ, R. A comprehensive review and evaluation of permutation flow shop heuristics to minimize flow time, Computers \& Industrial Engineering, v. 40, p. 117-128, 2013.

PINEDO, M. Scheduling: Theory, Algorithms, and Systems. Nova York: Springer, 2012. RAJENDRAN, C.; ZIEGLER, H. Heuristic for scheduling to minimize the sum of weighted flow time of jobs in a flow shop with sequence-dependent setup times of jobs. Production Planning and Control, v, 33, p. 281-284, 1997.

SOUZA, A. B. D.; MOCCELLIN, J. V. Meta-heurística híbrida algoritmo genéticobusca tabu para programação de operações flow shop. In: Simpósio Brasileiro de Pesquisa Operacional, 2000. Anais do XXXII SBPO, 2000, v. 32, p. 314-325.

SLACK, N.; CHAMBERS, S.; JOHNSTON, R. Administração da produção. São Paulo: Atlas, 1999.

TAHA, H. A. Pesquisa operacional. São Paulo: Pearson Prentice Hall, 2008.

VERGARA, S. C. Projeto e relatórios de pesquisa em administração. São Paulo: Atlas, 1998.

WANG, X.; CHENG, T. C. E. Two-machine flow shop scheduling with job class setups to minimize total flow time. Computers and Operations Research, v. 32, p. 2751-2770, 2005.

ZACCARELLI, S. B. Programação e controle da produção. São Paulo: Pioneira, 1987. 